\title{
Hydrosulfide adducts of organo-iridium anticancer complexes
}

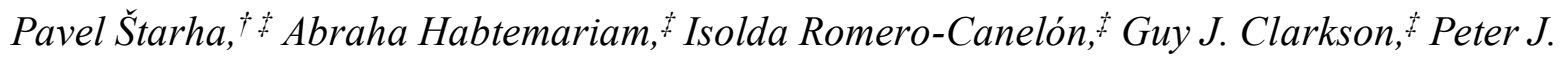
Sadler*,,+

†Regional Centre of Advanced Technologies and Materials, Department of Inorganic Chemistry, Faculty of Science, Palacký University, 17. listopadu 12, 77146 Olomouc, Czech Republic

Department of Chemistry, University of Warwick, Gibbet Hill Road, Coventry CV4 7AL, U.K.

\section{Supporting Information}

Synthesis of $\left[\left(\eta^{5}-C^{b i p h}\right) \operatorname{Ir}(\right.$ phen $\left.) C l\right] C l$ and $\left[\left(\eta^{5}-C^{b i p h}\right) \operatorname{Ir}(b p y) C l\right] C l$

\section{Tables S1 and S2}

Figures S1-S12 


\section{Synthesis $\left[\left(\eta^{5}-C p^{\text {biph }}\right) \operatorname{Ir}(\right.$ phen $\left.) \mathrm{Cl}\right] \mathrm{Cl}$ and $\left[\left(\eta^{5}-\mathbf{C} p^{\text {biph }}\right) \operatorname{Ir}(\right.$ bpy $\left.) C l\right] C l$}

Solutions of $\left[\left(\eta^{5}-\mathrm{Cp}^{\text {biph }}\right) \mathrm{IrCl}_{2}\right]_{2}(53.7 \mathrm{mg}, 0.05 \mathrm{mmol})$ and 1,10-phenanthroline $(21.6 \mathrm{mg}, 0.12 \mathrm{mmol})$ or 2,2-bipyridine $(18.7 \mathrm{mg}, 0.12 \mathrm{mmol})$ in $10 \mathrm{~mL}$ of methanol were heated for $1 \mathrm{~min}$ at $373 \mathrm{~K}$ in a microwave synthesizer. They were filtered and their volume was reduced to ca. $2 \mathrm{~mL}$ on a rotary evaporator. After cooling to ambient temperature, diethyl ether was added until light yellow precipitate formed. The products were collected by filtration, washed with diethyl ether and dried under vacuum (30 $\min )$.

$\left[\left(\eta^{5}-\mathrm{Cp}^{\text {biph }}\right) \operatorname{Ir}(\mathrm{phen}) \mathrm{Cl}\right] \mathrm{Cl}:$ Yield: $65 \mathrm{mg}(79 \%) .{ }^{1} \mathrm{H}$ NMR $\left(400 \mathrm{MHz}, \mathrm{DMSO}-d_{6}\right): \delta=9.12\left(\mathrm{~d}, 2 \mathrm{H}, \mathrm{J}_{\mathrm{HH}}\right.$ $=5.3 \mathrm{~Hz}), 8.99\left(\mathrm{~d}, 2 \mathrm{H}, \mathrm{J}_{\mathrm{HH}}=8.3 \mathrm{~Hz}\right), 8.39(\mathrm{~s}, 2 \mathrm{H}), 8.19\left(\mathrm{dd}, 2 \mathrm{H}, \mathrm{J}_{\mathrm{HH}}=8.2,5.4 \mathrm{~Hz}\right), 7.83\left(\mathrm{~d}, 2 \mathrm{H}, \mathrm{J}_{\mathrm{HH}}\right.$ $=8.0 \mathrm{~Hz}), 7.77\left(\mathrm{~d}, 2 \mathrm{H}, \mathrm{J}_{\mathrm{HH}}=7.5 \mathrm{~Hz}\right), 7.66\left(\mathrm{~d}, 2 \mathrm{H}, \mathrm{J}_{\mathrm{HH}}=8.3 \mathrm{~Hz}\right), 7.51\left(\mathrm{t}, 2 \mathrm{H}, \mathrm{J}_{\mathrm{HH}}=7.5 \mathrm{~Hz}\right), 7.42(\mathrm{t}$, $\left.1 \mathrm{H}, \mathrm{J}_{\mathrm{HH}}=7.5 \mathrm{~Hz}\right), 1.85(\mathrm{~s}, 6 \mathrm{H}), 1.77(\mathrm{~s}, 6 \mathrm{H})$.

$\left[\left(\eta^{5}-\mathrm{Cp}^{\text {biph }}\right) \operatorname{Ir}(\mathrm{bpy}) \mathrm{Cl}\right] \mathrm{Cl}:$ Yield: $58 \mathrm{mg}(73 \%) .{ }^{1} \mathrm{H}$ NMR $\left(400 \mathrm{MHz}, \mathrm{DMSO}-d_{6}\right): \delta=8.85\left(\mathrm{~d}, 2 \mathrm{H}, \mathrm{J}_{\mathrm{HH}}=\right.$ $8.3 \mathrm{~Hz}), 8.75\left(\mathrm{~d}, 2 \mathrm{H}, \mathrm{J}_{\mathrm{HH}}=5.8 \mathrm{~Hz}\right), 8.36\left(\mathrm{t}, 2 \mathrm{H}, \mathrm{J}_{\mathrm{HH}}=7.8 \mathrm{~Hz}\right), 7.84(\mathrm{~m}, 4 \mathrm{H}), 7.77\left(\mathrm{~d}, 2 \mathrm{H}, \mathrm{J}_{\mathrm{HH}}=7.5\right.$ $\mathrm{Hz}), 7.60\left(\mathrm{~d}, 2 \mathrm{H}, \mathrm{J}_{\mathrm{HH}}=8.3 \mathrm{~Hz}\right), 7.50(\mathrm{~m}, 2 \mathrm{H}), 7.42(\mathrm{~m}, 1 \mathrm{H}), 1.78(\mathrm{~s}, 6 \mathrm{H}), 1.72(\mathrm{~s}, 6 \mathrm{H})$. 
Table S1. Crystal data and structure refinements for $\left[\left(\eta^{5}-\mathrm{Cp} *\right) \operatorname{Ir}(\mathrm{phen})(\mathrm{SH})\right] \mathrm{BPh}_{4}\left(\mathbf{1}^{\prime}\right)$

\begin{tabular}{|c|c|}
\hline Empirical formula & $\mathrm{C}_{46} \mathrm{H}_{44} \mathrm{BIrN}_{2} \mathrm{~S}$ \\
\hline Formula weight & 859.90 \\
\hline Temperature (K) & $150(2)$ \\
\hline Crystal size (mm) & $0.6 \times 0.3 \times 0.3$ \\
\hline Wavelength $(\AA)$ & 0.71073 \\
\hline Crystal system, space group & Triclinic, $\mathrm{P}-1$ \\
\hline \multicolumn{2}{|l|}{ Unit cell dimensions } \\
\hline$a(\AA)$ & $11.3760(2)$ \\
\hline$b(\AA)$ & $15.3329(3)$ \\
\hline$c(\AA)$ & $22.0650(4)$ \\
\hline$\alpha\left(^{\circ}\right)$ & $101.801(2)$ \\
\hline$\beta\left(^{\circ}\right)$ & $93.536(2)$ \\
\hline$\gamma\left({ }^{\circ}\right)$ & $98.435(2)$ \\
\hline$V\left(\AA^{3}\right)$ & $3709.90(12)$ \\
\hline$Z, D_{\text {calc }}\left(\mathrm{g} \mathrm{cm}^{-3}\right)$ & $4,1.540$ \\
\hline Absorption coefficient $\left(\mathrm{mm}^{-1}\right)$ & 3.691 \\
\hline$F(000)$ & 1728 \\
\hline$\theta$ range for data collection $\left(^{\circ}\right)$ & $4.698 \leq 2 \theta \leq 61.322$ \\
\hline Reflections collected/unique & $154705 / 21878$ \\
\hline$R_{\text {int }}, R_{\text {sigma }}$ & $0.1445,0.1366$ \\
\hline Final $R$ indices $[I>2 \sigma(I)]$ & $R_{1}=0.0719$ \\
\hline$R$ indices (all data) & $\mathrm{w} R_{2}=0.1810$ \\
\hline Goodnes-of-fit & 1.010 \\
\hline
\end{tabular}


Table S2. Selected bond lengths $(\AA)$ and angles $\left(^{\circ}\right)$ of $\left[\left(\eta^{5}-\mathrm{Cp}^{*}\right) \operatorname{Ir}(\mathrm{phen})(\mathrm{SH})\right] \mathrm{BPh}_{4}(\mathbf{1})$ determined by a single-crystal X-ray analysis. Data are given for disordered $\left[\left(\eta^{5}-\mathrm{Cp} *\right) \operatorname{Ir}(\mathrm{phen})(\mathrm{SH})\right]^{+}$cation found in the asymmetric unit of $\mathbf{1}$ (i.e. cation involving Ir2 and Ir2A atoms)

\begin{tabular}{llll}
\hline \multicolumn{2}{l}{ Bond lengths $(\AA)$} & \multicolumn{2}{l}{ Bond angles $\left(^{\circ}\right)$} \\
\hline Ir-N & $2.107(7) / 2.28(3)$ & $\mathrm{N}-\mathrm{Ir}-\mathrm{N}$ & $76.6(3) / 70.1(12)$ \\
& $2.130(10) / 1.98(3)$ & $\mathrm{N}-\mathrm{Ir}-\mathrm{Cg}$ & $132.2(2) / 137.1(9)$ \\
$\mathrm{Ir}-\mathrm{C}^{\mathrm{a}}$ & $2.213(10) / 2.08(2)$ & & $131.6(2) / 123.6(8)$ \\
& $2.183(11) / 2.15(2)$ & $\mathrm{N}-\mathrm{Ir}-\mathrm{S}$ & $84.1(2) / 83.6(9)$ \\
& $2.150(12) / 2.10(2)$ & & $88.7(3) / 85.4(7)$ \\
& $2.181(12) / 1.99(2)$ & $\mathrm{S}-\mathrm{Ir}-\mathrm{Cg}$ & $126.16(8) / 133.6(2)$ \\
& $2.213(10) / 1.975(11)$ & & \\
$\mathrm{Ir}-\mathrm{Cg}{ }^{\mathrm{b}}$ & $1.8247(8) / 1.664(5)$ & & \\
$\mathrm{Ir}-\mathrm{S}$ & $2.384(3) / 2.428(5)$ & & \\
\hline
\end{tabular}

a) aromatic carbon atoms of $\mathrm{Cp} *$ ring

b) $\mathrm{Cg}=$ centroid of $\mathrm{Cp}^{*}$ aromatic ring 


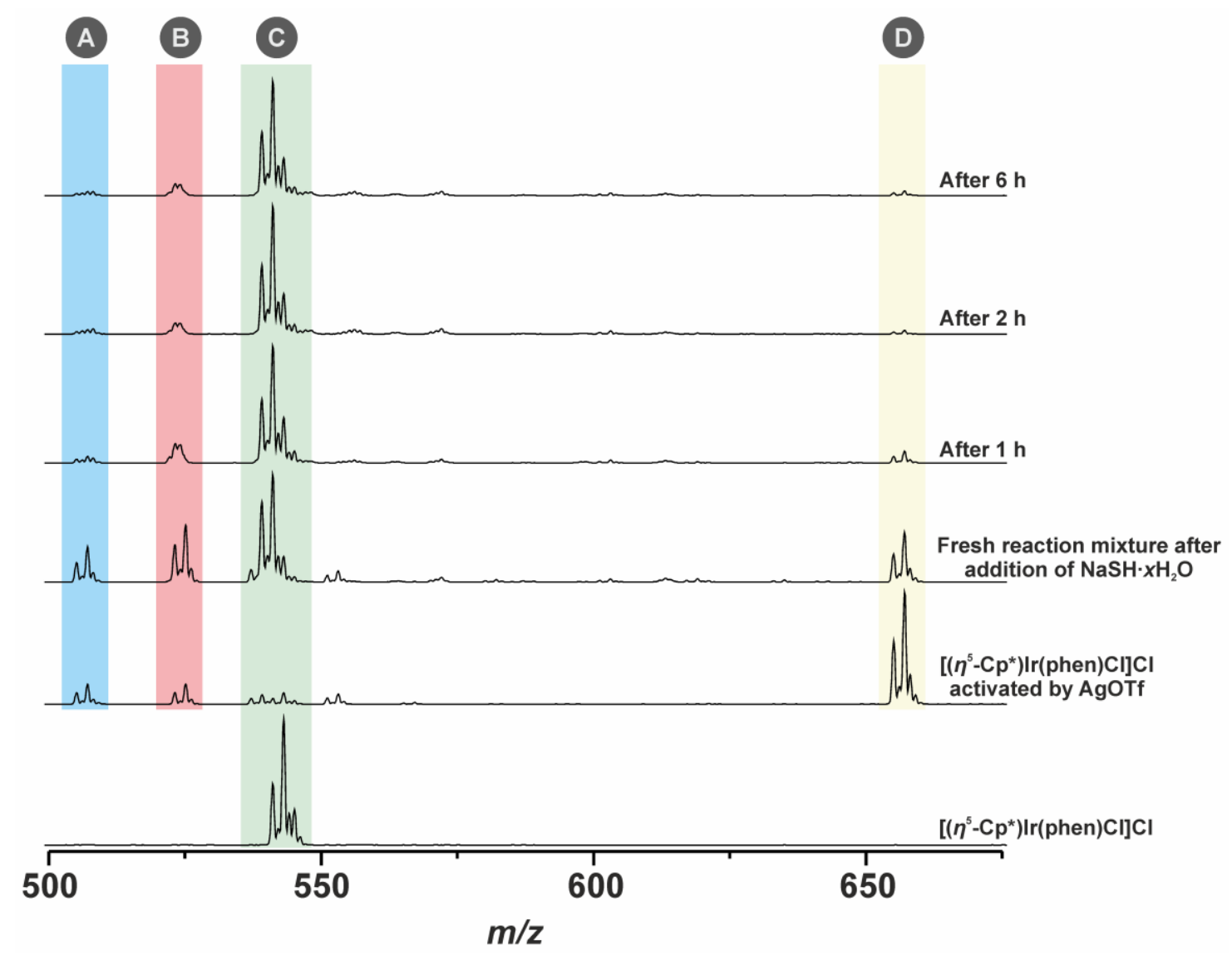

Figure S1. ESI-MS monitoring of the synthesis of 1: variation of the reaction time.

$\mathrm{A}=\left\{\left[\left(\eta^{5}-\mathrm{Cp} *\right) \operatorname{Ir}(\mathrm{phen})\right]-\mathrm{H}\right\}^{+}(m / z=507.2), \mathrm{B}=\left[\left(\eta^{5}-\mathrm{Cp}^{*}\right) \operatorname{Ir}(\mathrm{phen})(\mathrm{OH})\right]^{+}(m / z=525.2)$, $\mathrm{C}=\left[\left(\eta^{5}-\mathrm{Cp}^{*}\right) \operatorname{Ir}(\mathrm{phen})\left(\mathrm{OCH}_{3}\right)\right]^{+}(m / z=539.2),\left[\left(\eta^{5}-\mathrm{Cp}^{*}\right) \operatorname{Ir}(\mathrm{phen})(\mathrm{SH})\right]^{+}(m / z=541.1)$, $\left[\left(\eta^{5}-\mathrm{Cp}^{*}\right) \operatorname{Ir}(\mathrm{phen})(\mathrm{Cl})\right]^{+}(m / z=543.1)$ and $\mathrm{D}=\left[\left(\eta^{5}-\mathrm{Cp} *\right) \operatorname{Ir}(\mathrm{phen})(\mathrm{OTf})\right]^{+}(m / z=657.1)$. 


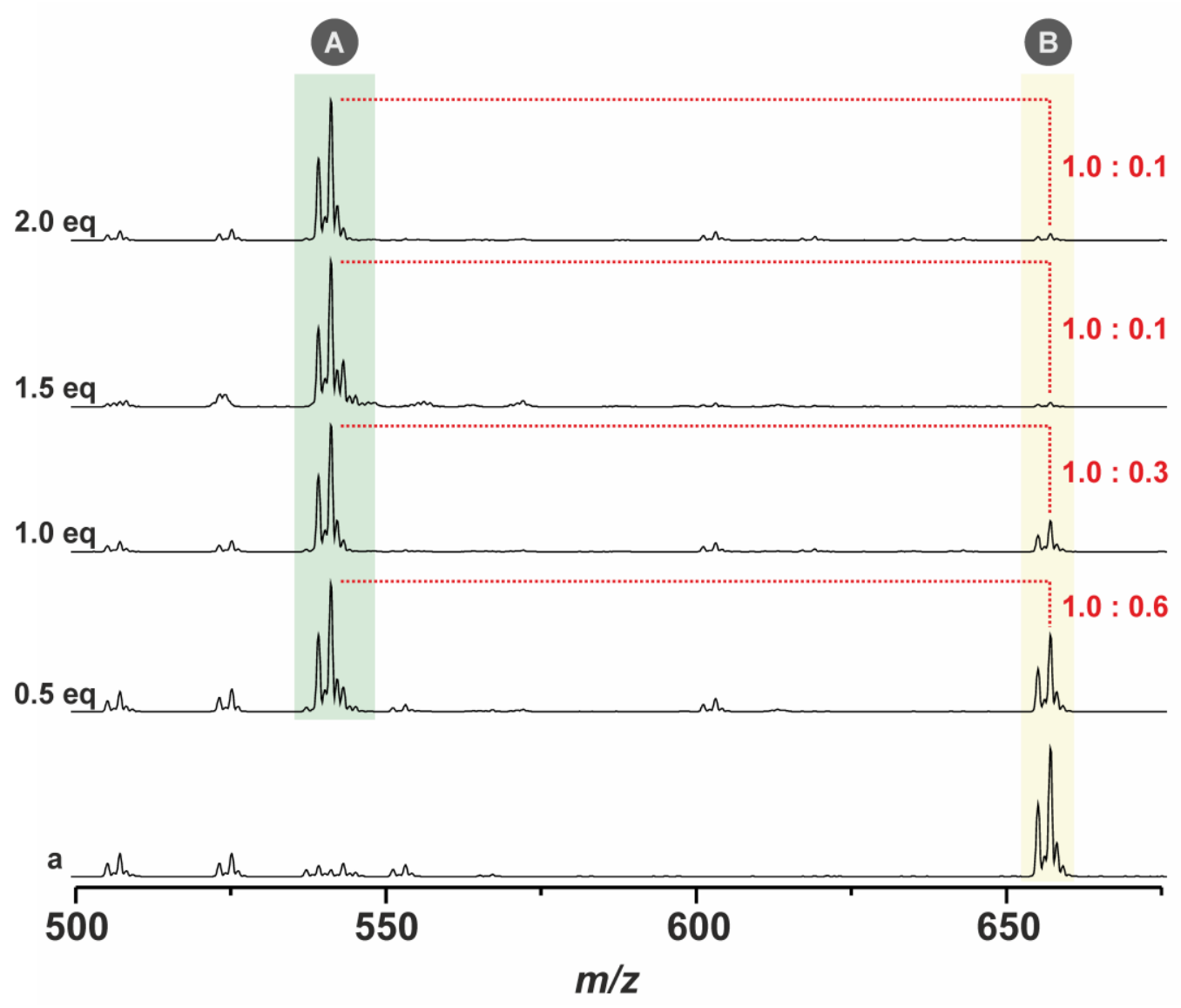

Figure S2. ESI-MS monitoring of the synthesis of 1: variation of the molar ratio of the starting compounds $\left[\left(\eta^{5}-\mathrm{Cp}^{*}\right) \mathrm{Ir}(\right.$ phen $\left.) \mathrm{Cl}\right] \mathrm{Cl}$ and $\mathrm{NaSH} \cdot x \mathrm{H}_{2} \mathrm{O}(0.5,1.0,1.5$ and $2.0 \mathrm{~mol}$ equiv). (a) shows $\left[\left(\eta^{5}-\mathrm{Cp}^{*}\right) \operatorname{Ir}(\mathrm{phen}) \mathrm{Cl}\right] \mathrm{Cl}$ activated by AgOTf. $\mathrm{A}=\left[\left(\eta^{5}-\mathrm{Cp}^{*}\right) \operatorname{Ir}(\mathrm{phen})(\mathrm{SH})\right]^{+}(m / z=541.1)$ and $\mathrm{B}=$ $\left[\left(\eta^{5}-\mathrm{Cp} *\right) \operatorname{Ir}(\mathrm{phen})(\mathrm{OTf})\right]^{+}(\mathrm{m} / z=657.1)$. 


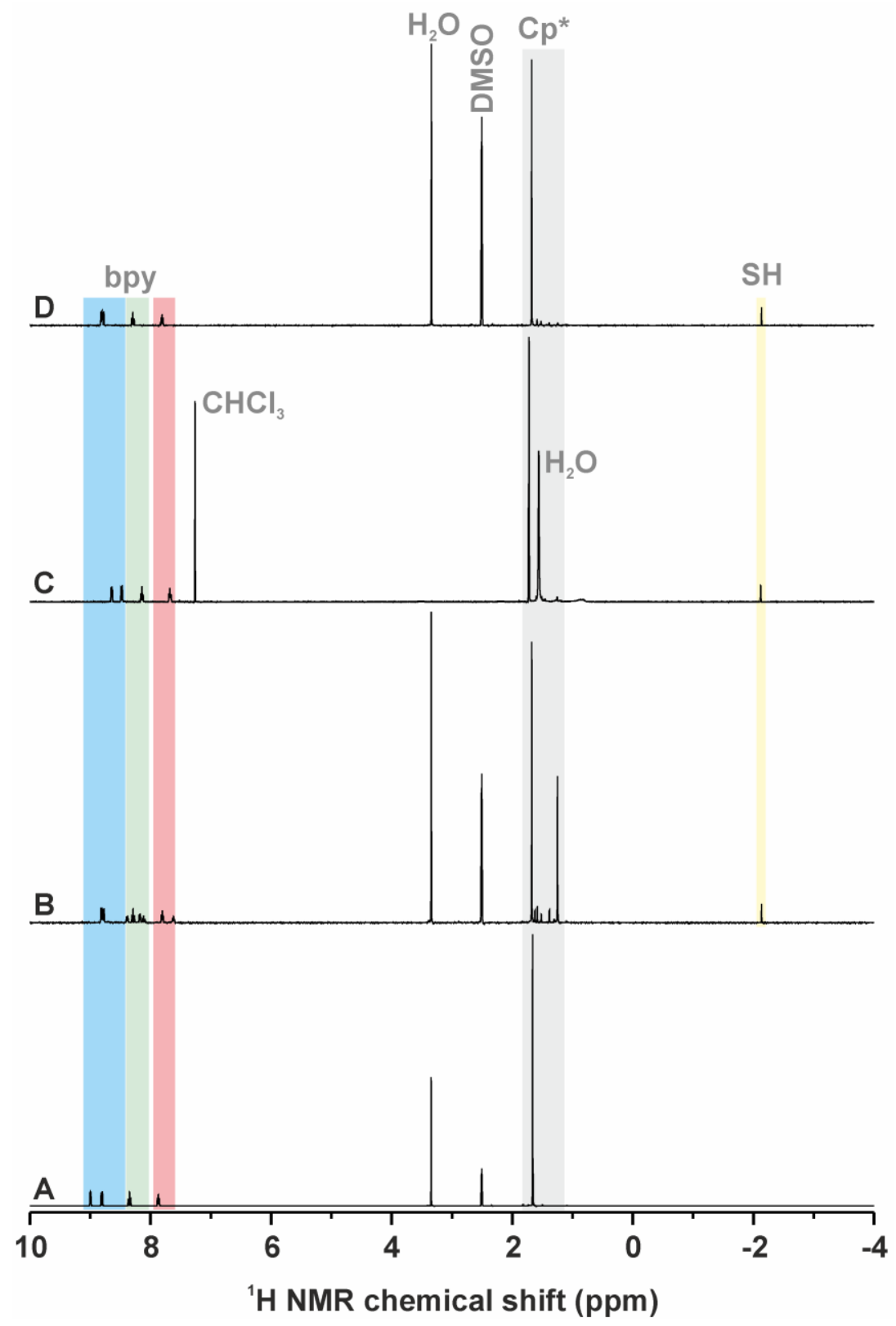

Figure S3. ${ }^{1} \mathrm{H}$ NMR spectra of the starting complex $\left[\left(\eta^{5}-\mathrm{Cp} *\right) \operatorname{Ir}(\mathrm{bpy}) \mathrm{Cl}\right] \mathrm{Cl}$ dissolved in DMSO- $d_{6}$ (A), the crude product $\left(2^{*}\right)$ dissolved in DMSO- $d_{6}(\mathrm{~B})$ and $\mathrm{CDCl}_{3}(\mathrm{C})$, and the final product, $\left[\left(\eta^{5}-\right.\right.$ $\mathrm{Cp} *) \operatorname{Ir}(\mathrm{bpy})(\mathrm{SH}) \mathrm{PF}_{6}(\mathbf{2})$, dissolved in $\mathrm{DMSO}-d_{6}(\mathrm{D})$. 

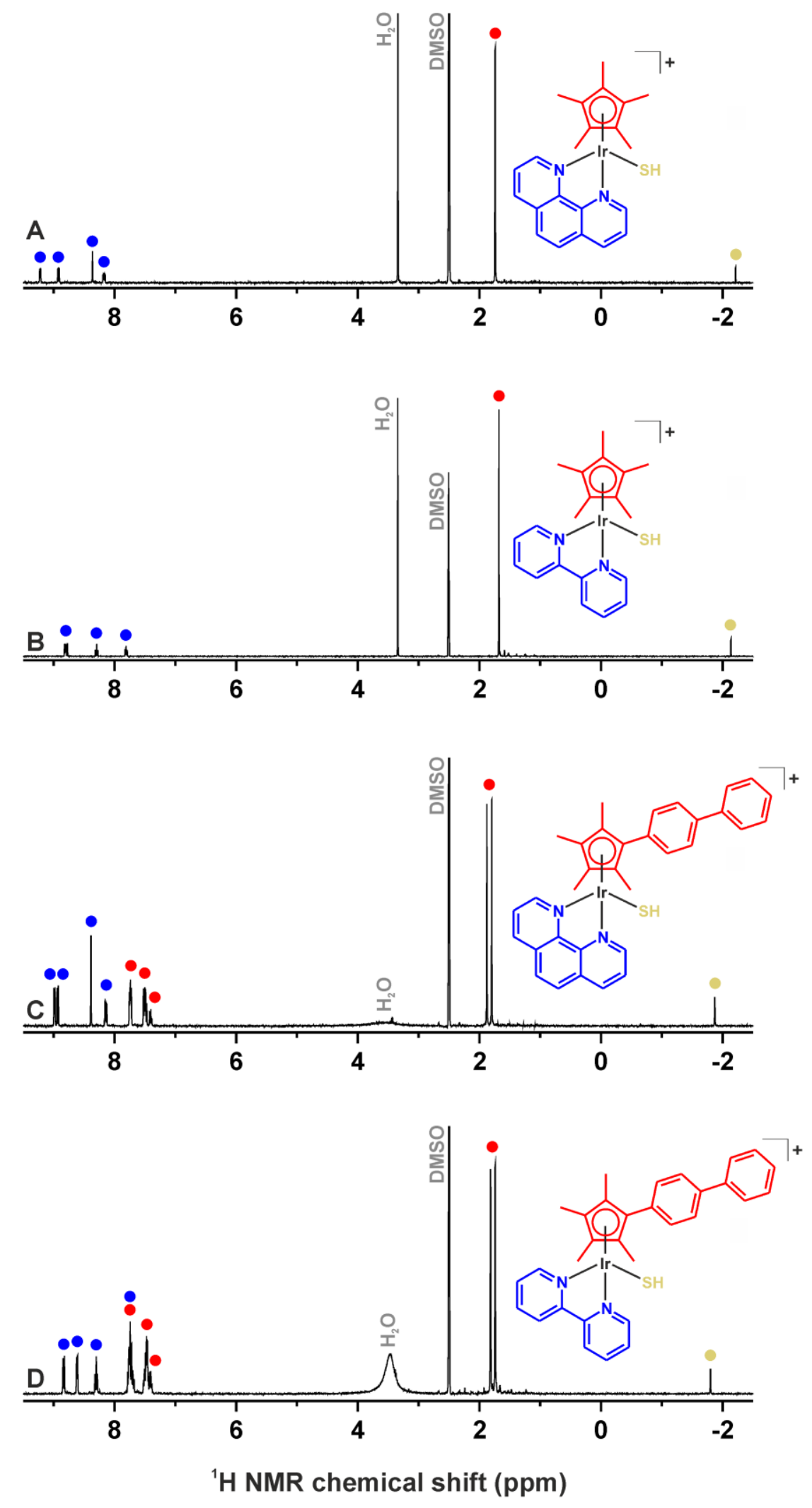

Figure S4. ${ }^{1} \mathrm{H}$ NMR spectra of $\left[\left(\eta^{5}-\mathrm{Cp}^{*}\right) \operatorname{Ir}(\mathrm{phen})(\mathrm{SH})\right] \mathrm{PF}_{6}(\mathbf{1} ; \mathrm{A}),\left[\left(\eta^{5}-\mathrm{Cp} \mathrm{p}^{*}\right) \operatorname{Ir}(\mathrm{bpy})(\mathrm{SH})\right] \mathrm{PF}_{6}(\mathbf{2} ; \mathrm{B})$, $\left[\left(\eta^{5}-\mathrm{Cp}^{\mathrm{biph}}\right) \operatorname{Ir}(\mathrm{phen})(\mathrm{SH})\right] \mathrm{PF}_{6}(\mathbf{3} ; \mathrm{C}),\left[\left(\eta^{5}-\mathrm{C} \mathrm{p}^{\mathrm{biph}}\right) \operatorname{Ir}(\mathrm{bpy})(\mathrm{SH})\right] \mathrm{PF}_{6}(\mathbf{4} ; \mathrm{D})$ dissolved in DMSO- $d_{6}$. 

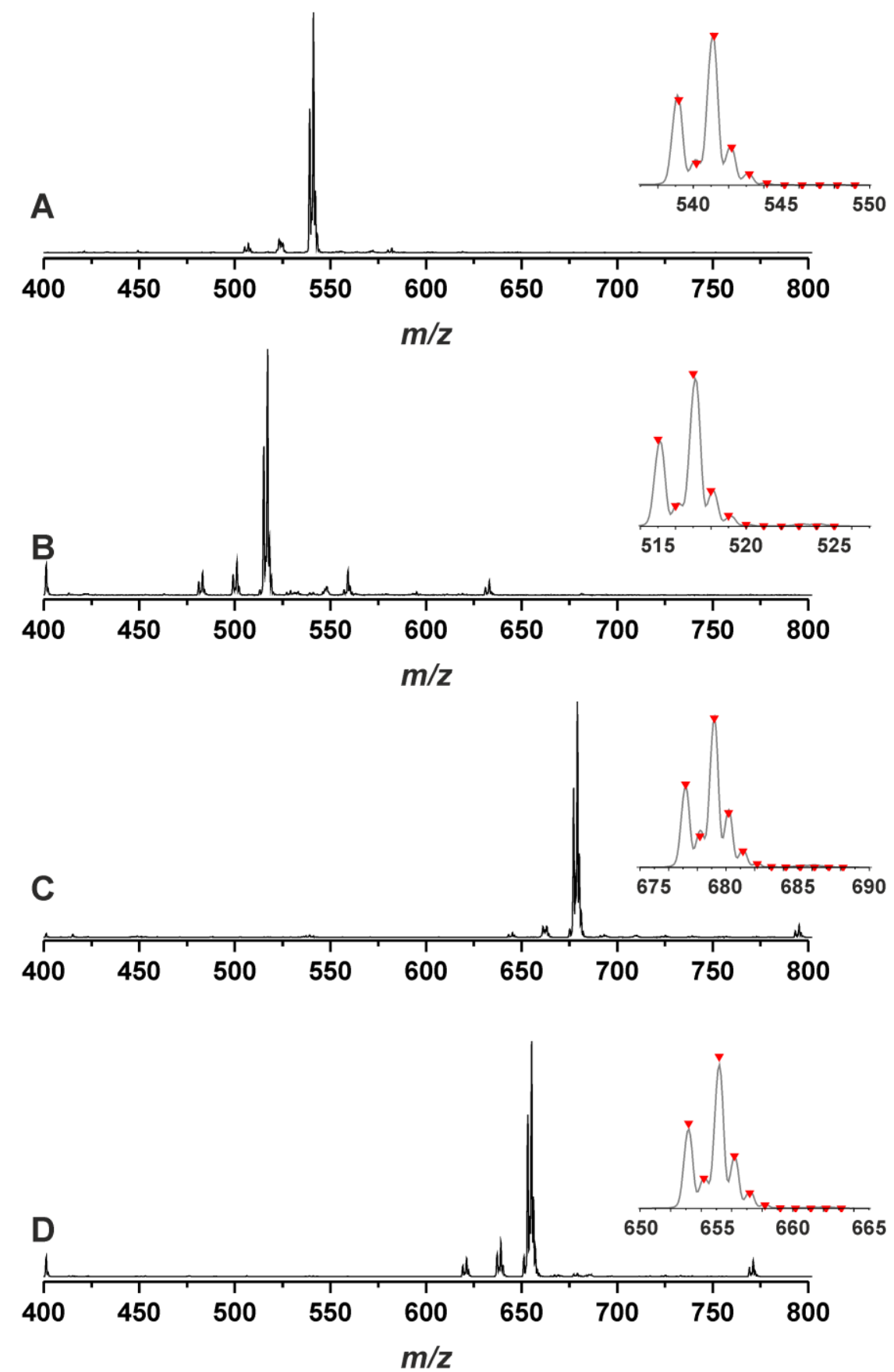

Figure S5. ESI-MS of $\left[\left(\eta^{5}-\mathrm{Cp}^{*}\right) \operatorname{Ir}(\mathrm{phen})(\mathrm{SH})\right] \mathrm{PF}_{6}(\mathbf{1} ; \mathrm{A}),\left[\left(\eta^{5}-\mathrm{Cp}^{*}\right) \operatorname{Ir}(\mathrm{bpy})(\mathrm{SH})\right] \mathrm{PF}_{6}(\mathbf{2} ; \mathrm{B}),\left[\left(\eta^{5}-\right.\right.$ $\left.\left.\mathrm{Cp}^{\text {biph }}\right) \operatorname{Ir}(\mathrm{phen})(\mathrm{SH})\right] \mathrm{PF}_{6}(3 ; \mathrm{C}),\left[\left(\eta^{5}-\mathrm{Cp}^{\mathrm{biph}}\right) \operatorname{Ir}(\mathrm{bpy})(\mathrm{SH})\right] \mathrm{PF}_{6}(4 ; \mathrm{D})$ dissolved in methanol, recorded in the 400-800 $\mathrm{m} / \mathrm{z}$ range. Insets: comparison of the experimental (gray lines) and simulated (red triangles) isotopic distribution of the $\left[\left(\eta^{5}-\mathrm{Cp}^{\mathrm{x}}\right) \operatorname{Ir}\left(\mathrm{N}^{\wedge} \mathrm{N}\right)(\mathrm{SH})\right]^{+}$species. 


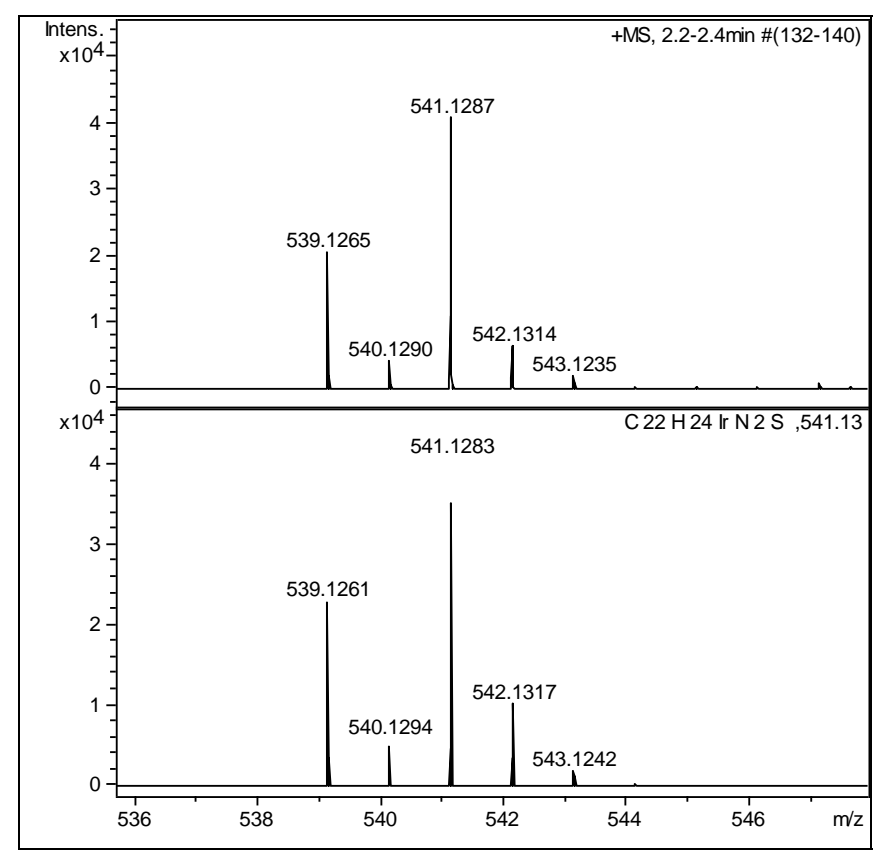

Figure S6. Experimental (up) and simulated (down) high resolution MS of [ $\left(\eta^{5}-\right.$ $\left.\mathrm{Cp}^{*}\right) \operatorname{Ir}(\mathrm{phen})(\mathrm{SH}) \mathrm{PF}_{6}(\mathbf{1} \mathrm{A})$ dissolved in methanol. 

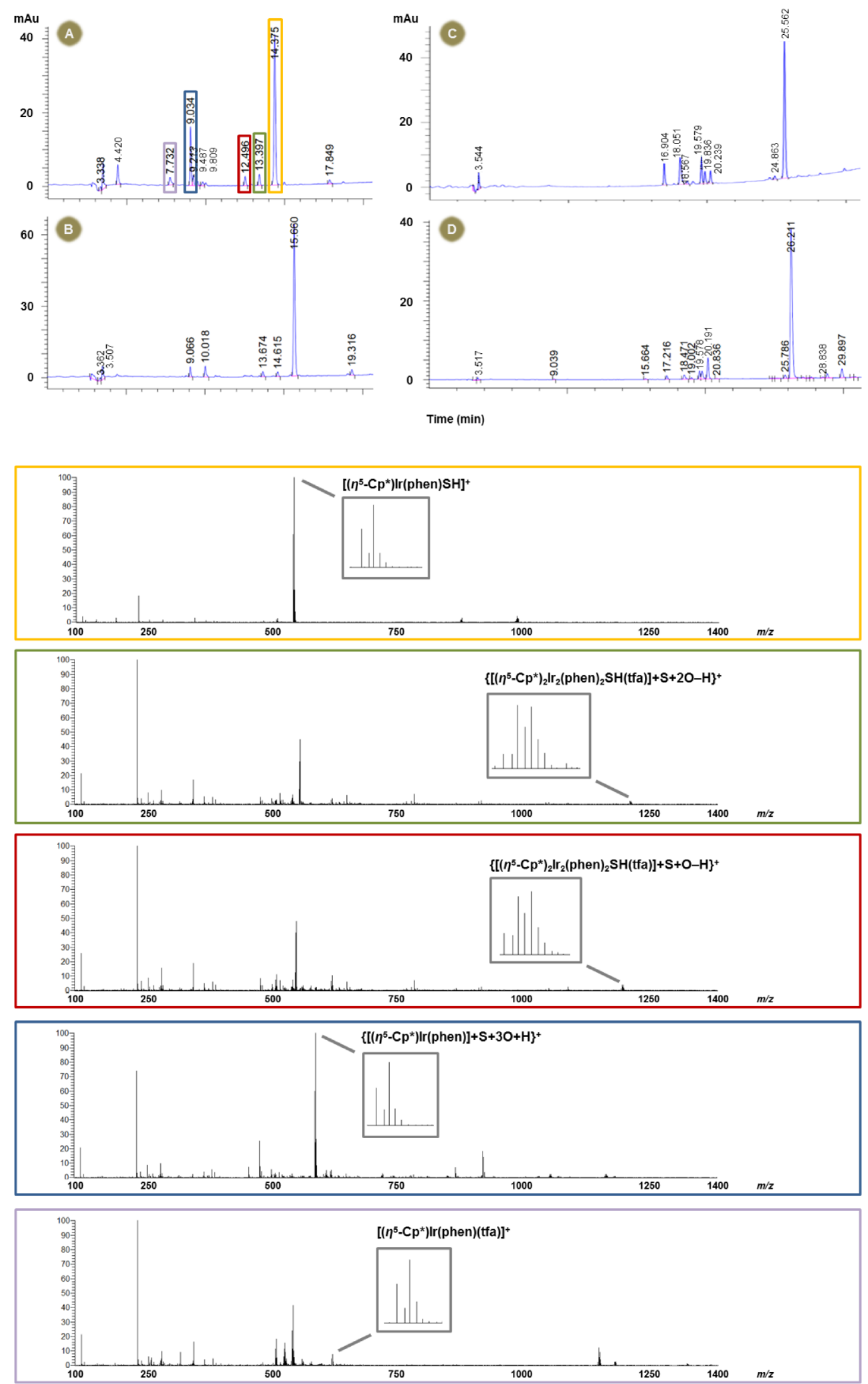

Figure S7. RP-HPLC chromatograms for $\left[\left(\eta^{5}-\mathrm{Cp} \mathrm{p}^{*}\right) \operatorname{Ir}(\mathrm{phen})(\mathrm{SH})\right] \mathrm{PF}_{6}(\mathbf{1} ; \mathrm{A}),\left[\left(\eta^{5}-\right.\right.$ $\left.\left.\mathrm{Cp}^{*}\right) \operatorname{Ir}(\mathrm{bpy})(\mathrm{SH})\right] \mathrm{PF}_{6}(\mathbf{2} ; \mathrm{B}),\left[\left(\eta^{5}-\mathrm{Cp}^{\mathrm{biph}}\right) \operatorname{Ir}(\mathrm{phen})(\mathrm{SH})\right] \mathrm{PF}_{6}(\mathbf{3} ; \mathrm{C})$ and $\left[\left(\eta^{5}-\mathrm{Cp}^{\mathrm{biph}}\right) \operatorname{Ir}(\mathrm{bpy})(\mathrm{SH})\right] \mathrm{PF}_{6}(\mathbf{4}$; D) given together with ESI-MS of the individual HPLC peaks for 1. 


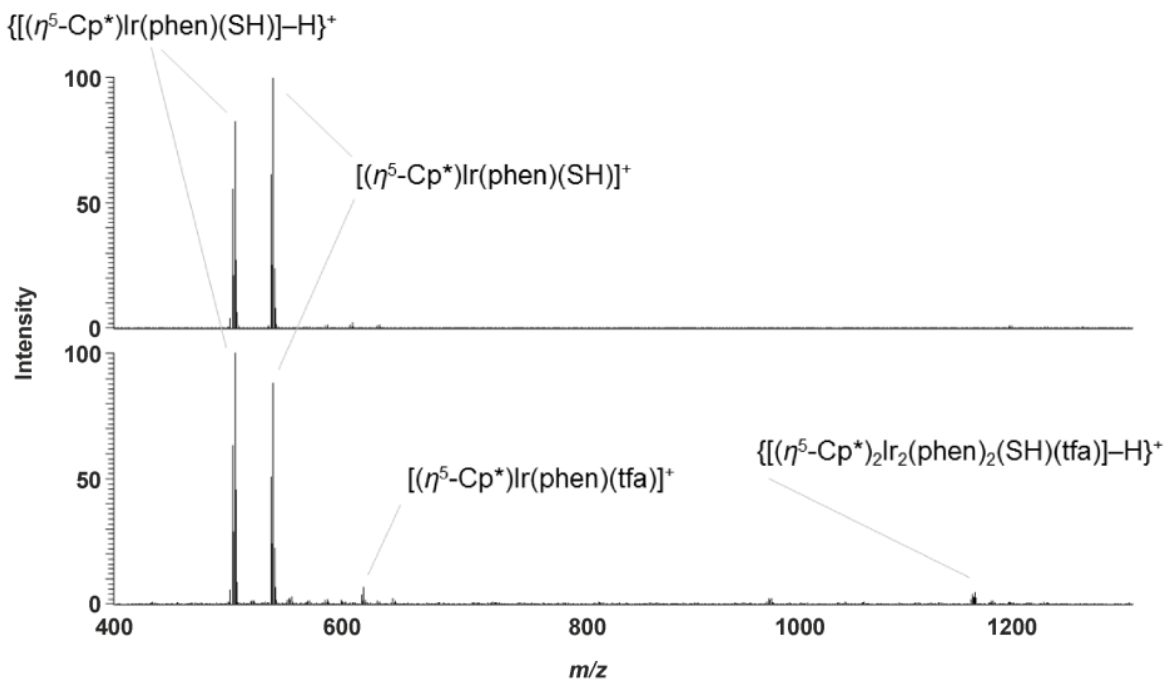

Figure S8. ESI-MS for $\left[\left(\eta^{5}-\mathrm{Cp} *\right) \operatorname{Ir}(\mathrm{phen})(\mathrm{SH})\right] \mathrm{PF}_{6}(\mathbf{1})$ dissolved in $\mathrm{H}_{2} \mathrm{O} / \mathrm{MeCN}$ (top) and in $\mathrm{H}_{2} \mathrm{O} / \mathrm{MeCN}$ with addition of $0.1 \% \mathrm{Htfa}$ (bottom) 


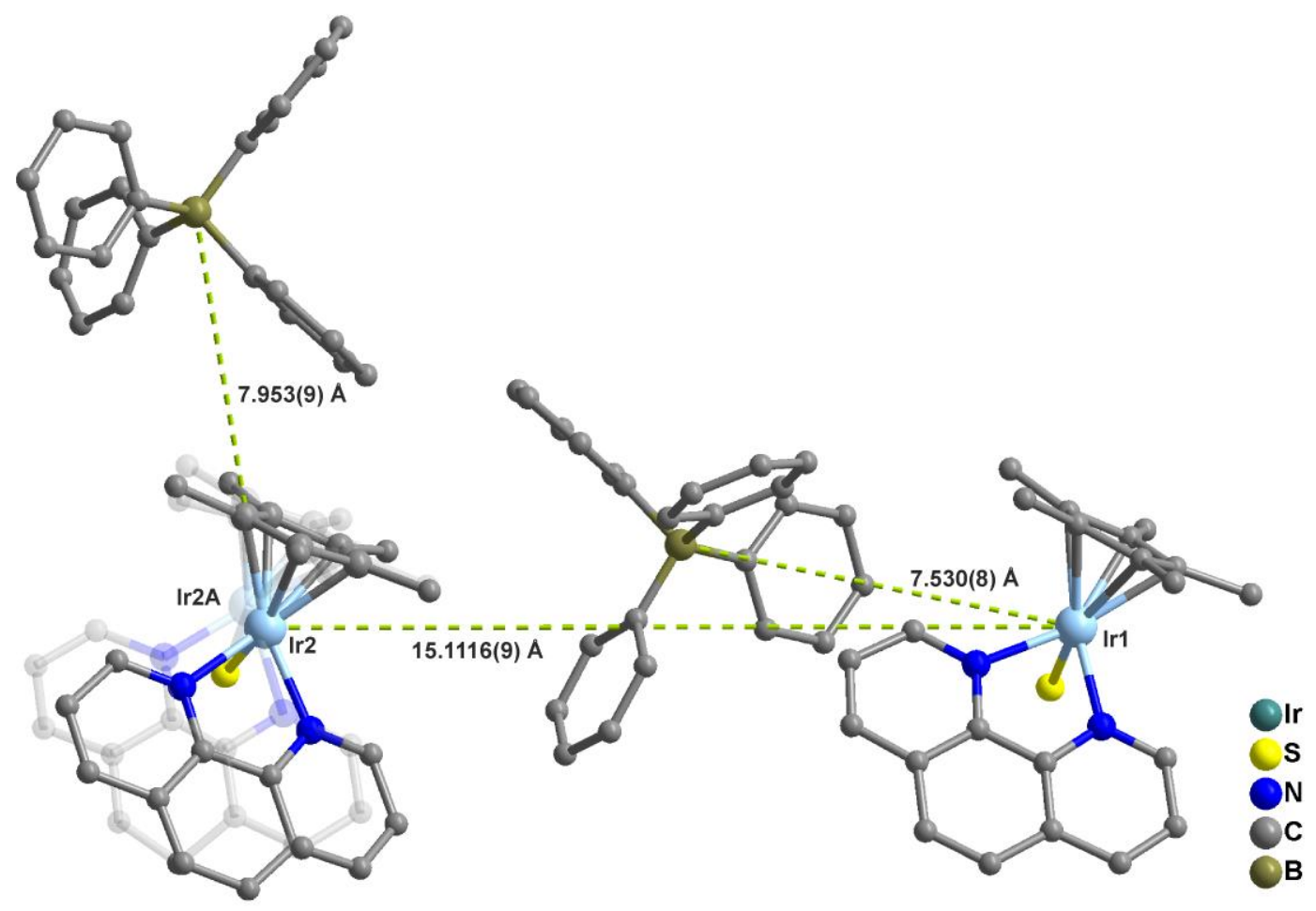

Figure S9. Part of the $\mathrm{x}$-ray crystal structure showing both $\left[\left(\eta^{5}-\mathrm{Cp} *\right) \operatorname{Ir}(\mathrm{phen})(\mathrm{SH})\right] \mathrm{BPh}_{4}$ molecules in the asymmetric unit of $\mathbf{1}^{-}$and disorder of the $\left[\left(\eta^{5}-\mathrm{Cp}^{*}\right) \operatorname{Ir}(\mathrm{phen})(\mathrm{SH})\right]^{+}$cation containing the $\operatorname{Ir} 2$ atom. 


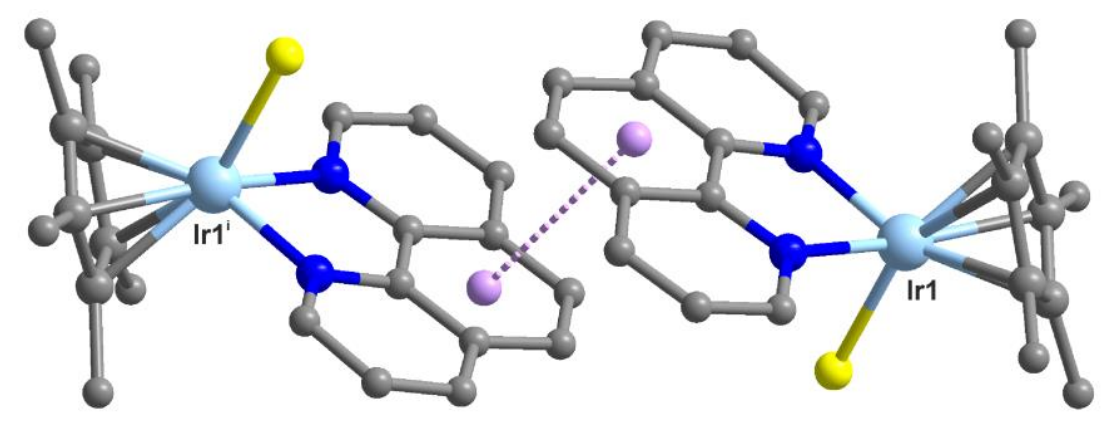

Figure S10. $\pi-\pi$ stacking in the $\mathrm{x}$-ray crystal structure of 1 between two adjacent phen ligands with a centroid-centroid distance of $3.4369 \AA$; symmetry code: i) 2-x, 2-y, 1-z. 


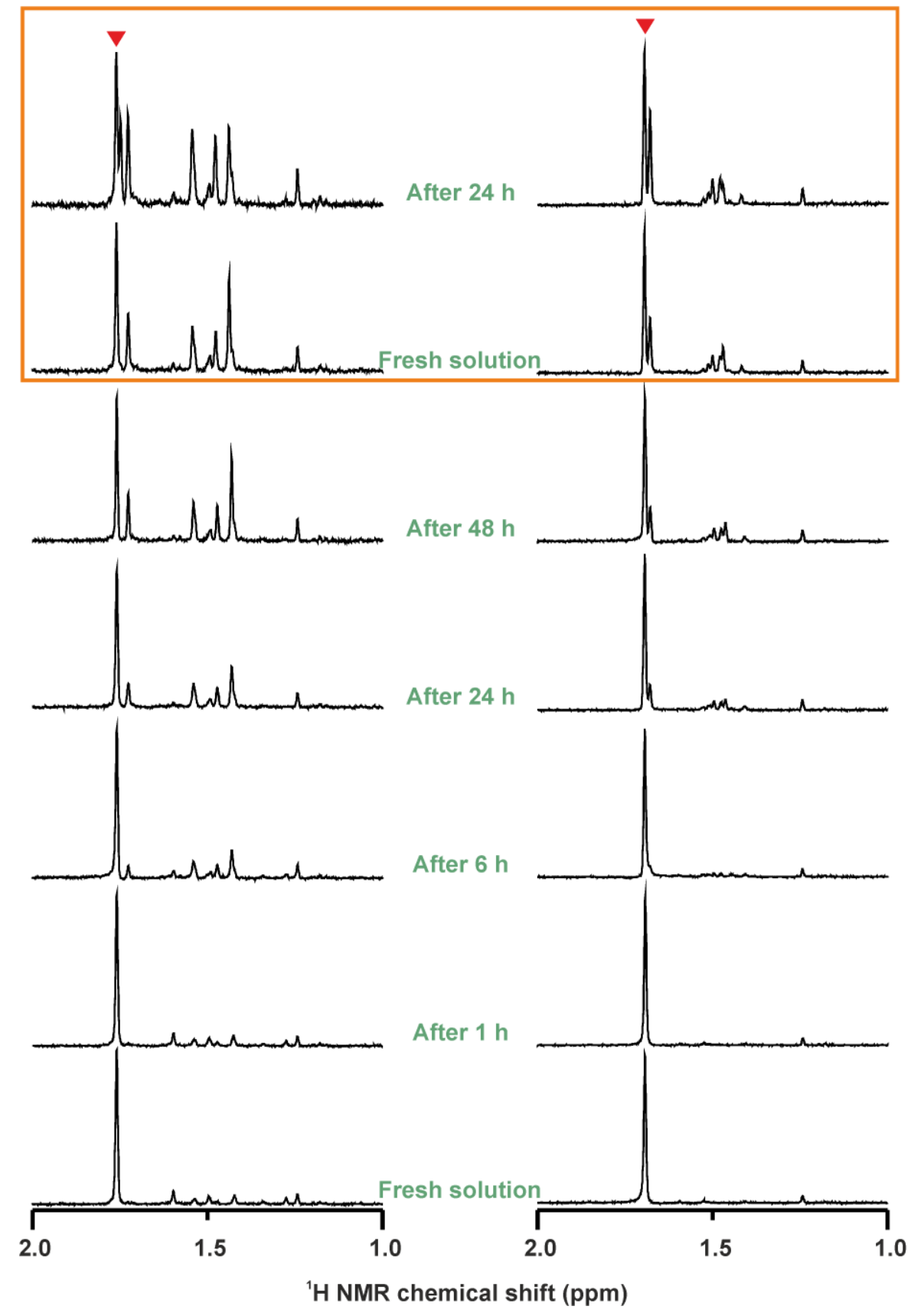

Figure S11. ${ }^{1} \mathrm{H}$ NMR spectra recorded at different times at $293 \mathrm{~K}$ for $0.5 \mathrm{mM}$ complexes $\left[\left(\eta^{5}\right.\right.$ $\left.\mathrm{Cp}^{*}\right) \operatorname{Ir}($ phen $\left.)(\mathrm{SH})\right] \mathrm{PF}_{6}(\mathbf{1}$; left $)$ and $\left[\left(\eta^{5}-\mathrm{Cp}^{*}\right) \operatorname{Ir}(\mathrm{bpy})(\mathrm{SH})\right] \mathrm{PF}_{6}(2 ;$ right $)$ dissolved in $20 \%$ $\mathrm{MeOD} / 80 \% \mathrm{D}_{2} \mathrm{O} .4 \mathrm{~mol}$ equiv of $\mathrm{NaCl}$ were added after $48 \mathrm{~h}$ (orange rectangle). 


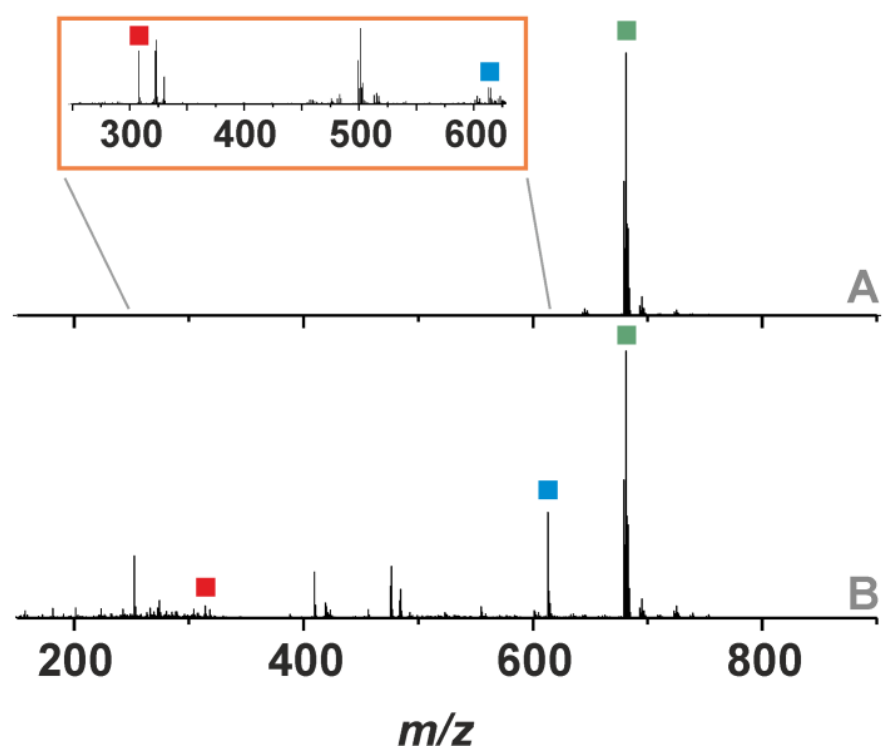

Figure S12. ESI-MS spectra of $\left[\left(\eta^{5}-\mathrm{Cp}^{\text {biph }}\right) \operatorname{Ir}(\mathrm{phen})(\mathrm{SH})\right] \mathrm{PF}_{6}(3)$ mixed with reduced glutathione $(\mathrm{GSH})$ in $20 \% \mathrm{MeOH} / 80 \% \mathrm{H}_{2} \mathrm{O}$. A - fresh solution; B - after $48 \mathrm{~h}$ of standing at ambient temperature. GSH - red squares, GSSG - blue squares, $\left[\left(\eta^{5}-\mathrm{Cp}^{\mathrm{biph}}\right) \operatorname{Ir}(\mathrm{phen})(\mathrm{SH})\right]^{+}$peaks - green squares. 